\title{
Forming and Quantifying Consensus in Distributed System Modeling and Group Decision-Making: A Perspective of Granular Computing
}

\author{
Witold Pedrycz ${ }^{(\bowtie)}$ \\ Department of Electrical and Computer Engineering, University of Alberta, \\ Edmonton AB T6R 2V4, Canada \\ wpedrycz@ualberta.ca
}

\begin{abstract}
In system modeling and decision-making, we encounter a variety of distributed sources of data that subsequently are transformed into a collection of sources of knowledge (viz. models and levels of preferences in case of group decision-making) producing an array of results. In general, these results differ from each other and therefore it becomes beneficial to build a consensus and quantify its quality. Furthermore the diversity of knowledge sources implies an inherent granular nature of the findings.

We advocate that the issues identified above can be formalized and supported by the formation of algorithmically sound solutions by engaging information granules (say, intervals, fuzzy sets, rough sets, etc.) and invoking the mechanisms of Granular Computing.

In this talk, we dwell upon the fundamental ideas and algorithms of Granular Computing such as the principle of justifiable granularity (and its generalizations) and an optimal allocation of information granularity. We show that the principle of justifiable granularity becomes instrumental in quantifying the diversity of individual sources of knowledge (say, in the form of fuzzy sets) and constructing information granules of type-2 (say, type-2 fuzzy sets). To endow the constructs with a required degree of flexibility, we admit some levels of information granularity across them by elevating their original numeric parameters to information granules.
\end{abstract}

